\title{
CRESCIMENTO RADICULAR E NUTRIÇÃO DE CULTIVARES DE ALGODOEIRO EM RESPOSTA À CALAGEM ${ }^{1}$
}

\author{
CIRO ANTONIO ROSOLEM ${ }^{2}$, GERSON SILVA GIOMMO ${ }^{3}$ e RENATO LUIS BENATTI LAURENTI ${ }^{3}$
}

\begin{abstract}
RESUMO - O crescimento radicular do algodoeiro é controlado geneticamente, mas fatores do solo como Al tóxico e baixos teores de Ca podem prejudicá-lo. Foi aplicado calcário a um Latossolo Vermelho-Escuro de textura média com $10 \%$ de saturação por bases, para se atingir 30,50 e $70 \%$ de saturação do solo por bases (usando-se quatro níveis de saturação), com o objetivo de estudar o crescimento radicular e a nutrição de três cultivares de algodão (Gossypium hirsutum L., v. latifolium Hutch): IAC 20, IAC 22 e CNPA-ITA 90, em resposta à calagem. O experimento foi conduzido em vasos com 4 L de solo, em condições de casa de vegetação, até 37 dias após a emergência das plantas, num delineamento fatorial $3 \times 4$, com quatro repetições em blocos casualisados. A calagem foi necessária para um bom crescimento radicular do algodoeiro. A cultivar IAC 22 mostrou sistema radicular mais tolerante à baixa saturação por bases, em comparação com IAC 20 e CNPA-ITA 90, mas o máximo crescimento radicular ocorreu em saturação por bases ao redor de 50\%, nas três cultivares. CNPA-ITA 90 foi mais exigente em K e mais tolerante ao Mn, com menor produção de matéria seca nas saturações mais altas.
\end{abstract}

Termos para indexação: Gossypium hirsutum, nutrientes, raízes, saturação do solo.

\section{ROOT GROWTH AND MINERAL NUTRITION OF COTTON CULTIVARS}

\section{AS AFFECTED BY LIMING}

\begin{abstract}
Cotton root growth is under genetic control but can be decreased by soil factors as Al toxicity and low Ca levels. Lime was applied to a Dark-Red Latosol (sandy loam) with $10 \%$ of base saturation, to raise base saturation to 30,50 and $70 \%$, in order to study the effects of liming on root growth and mineral nutrition of cotton (Gossypium hirsutum L., v. latifolium Hutch), cv. IAC 20, IAC 22 e CNPA-ITA 90 cultivares. The experiment was conducted in pots with $4 \mathrm{~L}$ of soil, under greenhouse conditions, up to 37 days after plant emergence in a $3 \times 4$ factorial design with four replications in randomized blocks. Liming was necessary in order to get a good cotton root growth. The IAC $22 \mathrm{cv}$. showed roots more tolerant to low soil base saturation than cv. IAC 20 and cv. CNPA-ITA 90. Maximum root growth was observed when the soil base saturation was around 50\%. CNPA-ITA 90 showed a higher $\mathrm{K}$ absorption and was more tolerant to high Mn levels, showing a decrease in dry matter production at the highest soil base saturation.
\end{abstract}

Index terms: Gossypium hirsutum, nutrients, roots, soil saturation.

\section{INTRODUÇÃO}

O sistema radicular do algodoeiro cresce em comprimento até a época de pleno florescimento. Após

${ }^{1}$ Aceito para publicação em 22 de abril de 1999.

${ }^{2}$ Eng. Agrôn., Dr., Prof. Titular, Dep. de Agricultura e Melhoramento Vegetal, Faculdade de Ciências Agronômicas, UNESP, Caixa Postal 237, CEP 18603-970 Botucatu, SP. Bolsista do CNPq. E-mail: rosolem@fca.unesp.br

${ }^{3}$ Eng. Agrôn., Curso de Pós-Graduação em Agricultura. Faculdade de Ciências Agronômicas, UNESP. esse período, existe apenas incremento na matéria seca das raízes (Nayakekorala \& Taylor, 1990). Esse sistema radicular é pouco volumoso, explorando pobremente certas regiões do solo, mas existem diferenças entre genótipos (Brouder \& Cassman, 1990).

Embora o padrão de enraizamento esteja sob controle genético, o crescimento das raízes é modificado por fatores químicos e físicos do solo (Taylor \& Arkin, 1981). A presença de Al tóxico prejudica muito o desenvolvimento radicular (Adams \& 
Pearson, 1970). Além disso, o crescimento radicular do algodoeiro foi inibido quando a saturação do complexo de troca do solo por $\mathrm{Ca}$ era menor que $17 \%$ (Adams \& Moore, 1983). Por outro lado, os teores de Ca no solo tidos como críticos para o crescimento radicular variam de 2,5 a 8,0 $\mathrm{mmol}_{\mathrm{c}} \mathrm{dm}^{-3}$ (Adams \& Moore, 1983), embora Rosolem et al. (1995) tenham encontrado resposta em crescimento de raízes de soja mesmo quando o solo apresentava originalmente um teor de 20,0 $\mathrm{mmol}_{\mathrm{c}} \mathrm{dm}^{-3} \mathrm{de} \mathrm{Ca}$.

Silva et al. (1995) demonstraram que as maiores produtividades da cv. IAC 20 somente foram observadas quando a camada de 0 a $20 \mathrm{~cm}$ de profundidade do solo apresentava aproximadamente $60 \%$ de saturação por bases. Além disso, a correção subsuperficial, ou seja, a elevação da saturação do solo por bases a aproximadamente $40 \%$ até $60 \mathrm{~cm}$ de profundidade, concorreu para a boa produção do algodoeiro.

Por outro lado, Rosolem et al. (1998) observaram que o aumento da saturação do solo por bases até $52 \%$ foi efetivo em prevenir o decréscimo de crescimento radicular do algodão causado pela compactação do solo. Entretanto, quando efetuaram calagem para elevar a saturação do solo por bases a $66,7 \%$, notaram decréscimo no crescimento radicular do algodoeiro IAC 20, atribuindo tal efeito a uma possível deficiência de $\mathrm{Zn}$ induzida pela calagem.

Estudando a interação calagem $\mathrm{x}$ adubação fosfatada em milho, Rosolem et al. (1994) verificaram que ocorreu aumento na superfície radicular em função da calagem somente nos tratamentos que não receberam P. Acontece que a fonte de P utilizada foi o superfosfato triplo, que contém Ca que, no caso, foi suficiente para promover o crescimento radicular. Assim, em estudos do efeito da calagem sobre o sistema radicular é importante o emprego de uma fonte de $\mathrm{P}$ isenta de Ca para que o resultado não seja mascarado.

Considerando que as recomendações de calagem no Estado de São Paulo foram desenvolvidas com base em resultados obtidos principalmente com a cv. IAC 20, e que podem existir diferenças genéticas, o presente trabalho objetivou estudar a resposta à calagem, o crescimento radicular e a nutrição, comparando três cultivares de algodoeiro durante a fase de estabelecimento da cultura.

\section{MATERIAL E MÉTODOS}

Foi realizado um experimento na Faculdade de Ciências Agronômicas, UNESP, em Botucatu, SP, em vasos de cerâmica revestida internamente com tinta betuminosa, com $4 \mathrm{~L}$ de material de solo proveniente da camada arável de um Latossolo Vermelho-Escuro de textura média, em ambiente de casa de vegetação.

Antes do início dos tratamentos, a análise química das amostras de material de solo (Raij \& Quaggio, 1983) revelou $\mathrm{pH} 4,0 ; 11 \mathrm{~g} \mathrm{~kg}^{-1}$ de $\mathrm{MO} ; 6 \mathrm{mg} \mathrm{kg}{ }^{-1} \mathrm{de}$ $\mathrm{P} ; 34 \mathrm{mmol}_{\mathrm{c}} \mathrm{dm}^{-3}$ de $\mathrm{H}+\mathrm{Al} ; 0,9 \mathrm{mmol}_{\mathrm{c}} \mathrm{dm}^{-3}$ de $\mathrm{K}$; 2,0 $\mathrm{mmol}_{\mathrm{c}} \mathrm{dm}^{-3} \mathrm{de} \mathrm{Ca} ; 1,0 \mathrm{mmol}_{\mathrm{c}} \mathrm{dm}^{-3} \mathrm{de} \mathrm{Mg} ; 4,0 \mathrm{mmol}_{\mathrm{c}} \mathrm{dm}^{-3}$ de $\mathrm{SB}$ e $38 \mathrm{mmol}_{\mathrm{c}} \mathrm{dm}^{-3}$ de CTC, com saturação por bases de $10 \%$. Foi realizada calagem com calcário dolomítico calcinado (PRNT=105), em doses calculadas para se atingir 30, 50 e $70 \%$ de saturação por bases. A terra dos vasos foi posta em incubação úmida, com umidade de aproximadamente $80 \%$ da capacidade de retenção de água do solo, por 30 dias. Após esse período foi realizada adubação com $150 \mathrm{mg} \mathrm{kg}^{-1}$ de $\mathrm{P}$ como $\mathrm{NH}_{4} \mathrm{H}_{2} \mathrm{PO}_{4}$ e $\mathrm{K}_{2} \mathrm{HPO}_{4}, 120 \mathrm{mg} \mathrm{kg}^{-1}$ de $\mathrm{K}$ como cloreto de potássio, $24 \mathrm{mg} \mathrm{kg}^{-1}$ de $\mathrm{N}$ como sulfato de amônio e 1,0 $\mathrm{mg} \mathrm{kg}^{-1}$ de $\mathrm{B}$ como bórax, que foram misturados ao solo dos vasos. Aos 16 dias após a emergência das plantas, foram aplicados, em cobertura, $50 \mathrm{mg} \mathrm{kg}^{-1}$ de $\mathrm{N}$ na forma de uréia.

Foram semeadas cinco sementes por vaso de cada uma das cultivares IAC 20, IAC 22 e CNPA-ITA 90. Uma semana após a emergência, foi realizado o desbaste, deixando-se duas plantas por vaso, que foram cultivadas até 37 dias após a emergência.

$\mathrm{Na}$ colheita do experimento, as raízes foram separadas do solo por lavagem em água corrente sobre peneira de $0,5 \mathrm{~mm}$. Foi tomada uma amostra de aproximadamente $30 \%$ do sistema radicular, e se determinou o comprimento (Tennant, 1975), raio e superfície (Hallmark \& Barber, 1984). O restante do sistema radicular, assim como a parte aérea das plantas, foi secado em estufa com circulação forçada de ar, a $65^{\circ} \mathrm{C}$, por três dias, pesado e moído. Amostras dos tecidos foram digeridas por via úmida com a mistura nitroperclórica e foram analisados o $\mathrm{P}$ pelo método do vanado- 
molibdato, o K por espectrometria de emissão, e $\mathrm{Ca}, \mathrm{Mg}$, $\mathrm{Mn}$ e $\mathrm{Zn}$ por espectrometria de absorção atômica. Para a análise de $\mathrm{B}$ foi feita incineração do tecido, e o nutriente foi determinado por colorimetria com Azometina-H.

$\mathrm{O}$ experimento foi instalado segundo um modelo fatorial com três cultivares e quatro saturações do solo por bases (três resultantes da aplicação de calcário, mais a original do solo), com quatro repetições. Foi realizada análise de variância dos resultados obtidos, separando-se as médias pelo teste $\mathrm{t}$ (LSD), a $5 \%$ de probabilidade. Onde apropriado, foi realizado estudo de regressões, escolhendo-se o modelo de melhor ajuste pelo maior valor do coeficiente da regressão.

\section{RESULTADOS E DISCUSSÃO}

A produção de matéria seca de raízes aumentou de forma quadrática, nas três cultivares, apresentando o máximo em torno da saturação por bases de $50 \%$ (Fig. 1). Esse resultado confirma a observação de Rosolem et al. (1998), que notou decréscimo no sistema radicular do algodão IAC 20 quando a saturação do solo por bases foi elevada de
52 para $67 \%$. Neste estudo, a cultivar IAC 20 sempre teve menor massa radicular que as demais. Por outro lado, a massa da parte aérea e a produção total das cultivares IAC 20 e IAC 22 apresentaram resposta positiva à calagem até $70 \%$ de saturação por bases, enquanto a CNPA-ITA 90 apresentou o máximo em torno de $40 \%$ de saturação, e produziu menor massa que as demais na saturação de $70 \%$ (Fig. 1). De fato, pelo menos para IAC 20 seria esperado esse tipo de resposta, de acordo com os resultados obtidos por Silva et al. (1995).

Não foram observados efeitos significativos dos tratamentos sobre o diâmetro das raízes secundárias, que foi, em média, 0,03 mm. Pela Fig. 2, nota-se que a cultivar IAC 22 se diferenciou das demais no crescimento radicular em comprimento e superfície, pois mostrou maior habilidade em crescer na menor saturação por bases, e apresentou resposta, em comprimento, até $70 \%$ de saturação por bases, enquanto IAC 20 e CNPA-ITA 90 apresentaram resposta quadrática com máximo em torno de $50 \%$ de
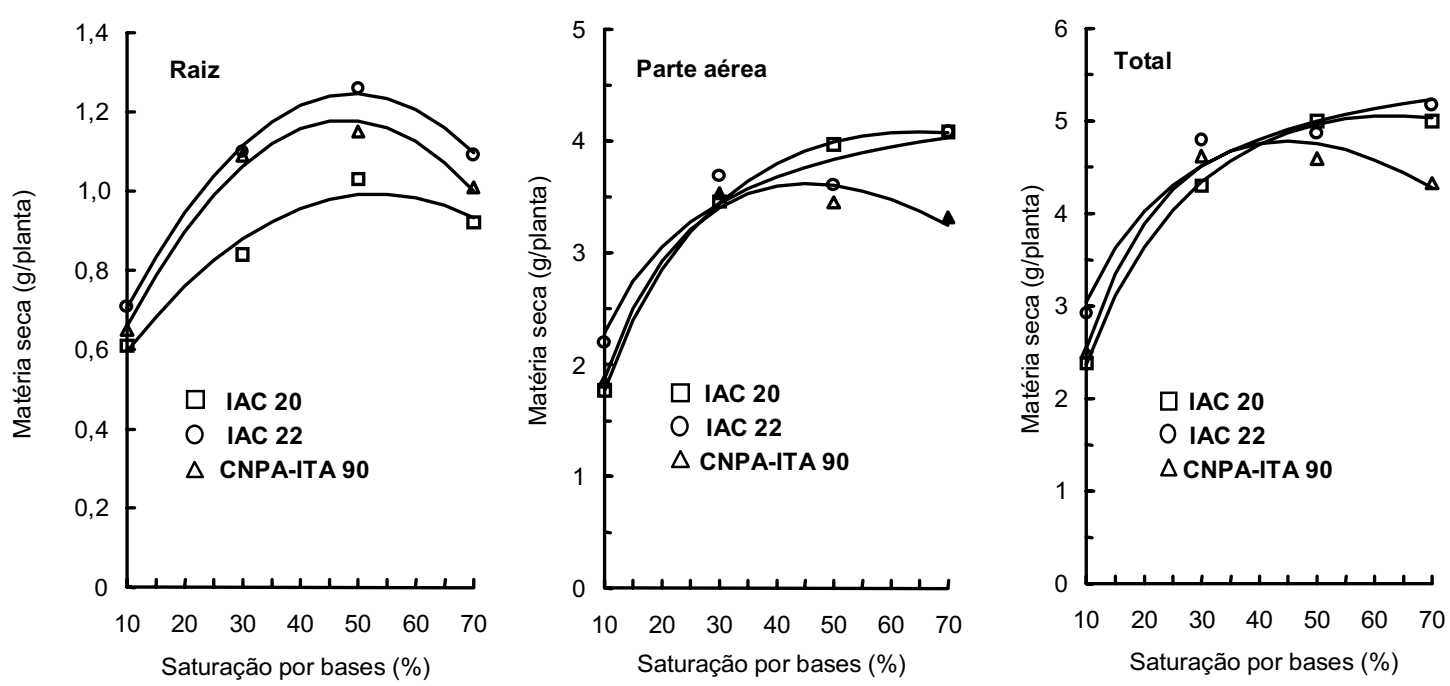

FIG. 1. Produção de matéria seca do algodoeiro em razão da cultivar e da saturação do solo por bases. Raiz: IAC $20-y=0,39+0,02 x-0,0002 x^{2}, R^{2}=0,98 ;$ IAC $22-y=0,39+0,03 x-0,0004 x^{2}, R^{2}=0,99$; CNPA-ITA $90-y=0,34+0,03 x-0,0004 x^{2}, R^{2}=0,99$. Parte aérea: IAC $20-y=2,19+1,56 x^{1 / 2}-0,097 x$, $R^{2}=0,99$; IAC $22-y=4,62 x /(10,3+x), R^{2}=0,95 ;$ CNPA-ITA $90-y=2,60+1,85 x^{1 / 2}-0,14 x, R^{2}=0,99$. Total: IAC $20-y=-2,34+1,86 x^{1 / 2}-0,117 x, R^{2}=0,99 ;$ IAC $22-y=5,95 x /(9,59+x), R^{2}=0,99$; CNPA-ITA $90-y=-3,3+2,4 x^{1 / 2}-0,18 x, R^{2}=0,99$. 
saturação. Nesse caso, os resultados obtidos para IAC 20 e CNPA-ITA 90 são semelhantes aos obtidos por Rosolem et al. (1998). Embora tenha sido observada diferença no padrão de resposta, não houve diferença significativa no comprimento e superfície das raízes, entre as cultivares, a partir da saturação de 30\% (Fig. 2). De modo geral, pode-se inferir que o sistema radicular de IAC 22 é menos sensível à variação da saturação do solo por bases que as demais cultivares.

Não houve efeito significativo da calagem nos teores de $\mathrm{P}, \mathrm{K}$ e Ca das raízes do algodoeiro. Entretanto, houve aumento nos teores de $\mathrm{Mg}$ e B, enquanto os teores de $\mathrm{Mn}$ e $\mathrm{Zn}$ foram diminuídos (Tabela 1). Com relação ao efeito das cultivares, somente foi notado efeito significativo para $\mathrm{Zn}, \mathrm{Mn}$ e $\mathrm{K}$ na raiz (Tabela 2). IAC 20 apresentou os menores teores de $\mathrm{Zn}$ e Mn, enquanto CNPA-ITA 90 apresentou o menor teor de $\mathrm{K}$.

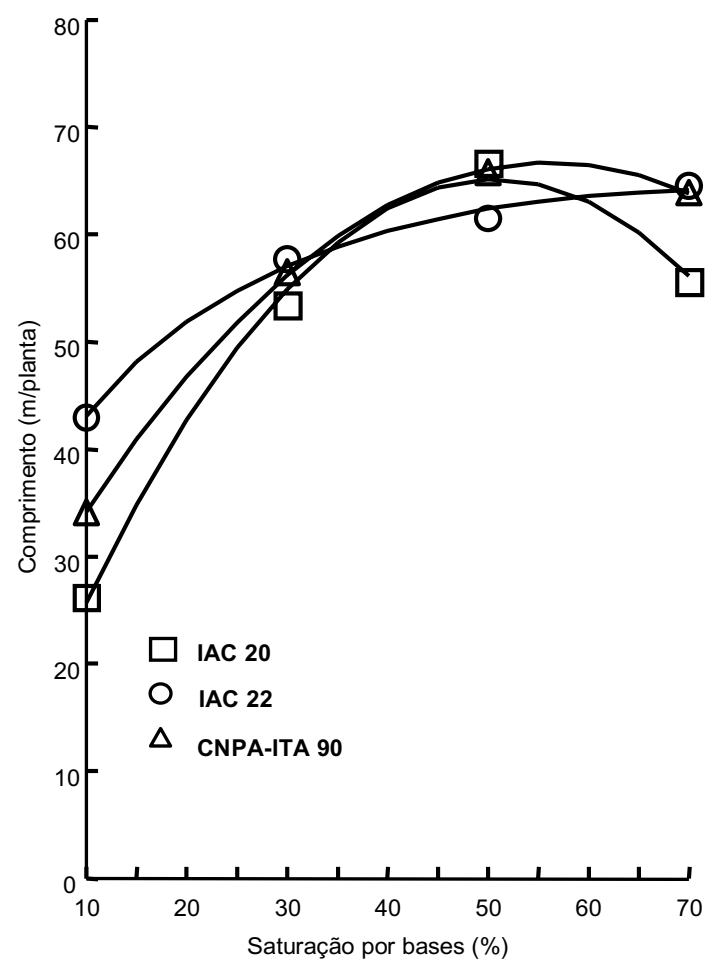

Rosolem et al. (1998) atribuíram o menor crescimento observado na maior saturação por bases a uma possível deficiência de Zn. Neste trabalho, a parte aérea das plantas não estava deficiente em $\mathrm{Zn}$, mas houve diminuição significativa nos teores desse nutriente nas raízes em função da calagem. Entretanto, o decréscimo observado não foi grande em termos absolutos, deixando dúvidas quanto ao seu efeito no crescimento radicular.

Com relação ao $\mathrm{P}$, foi observada interação dos efeitos das saturações por bases com as cultivares (Fig. 3), de modo que a cv. CNPA-ITA 90 demonstrou ser mais responsiva, pois na menor saturação apresentou menor teor que as demais, mas a situação se inverteu na maior saturação. Martini \& Mutters (1989) relataram que a calagem excessiva inibe o crescimento radicular, podendo até prejudicar a absorção de $\mathrm{P}$, o que não foi observado no presente trabalho.

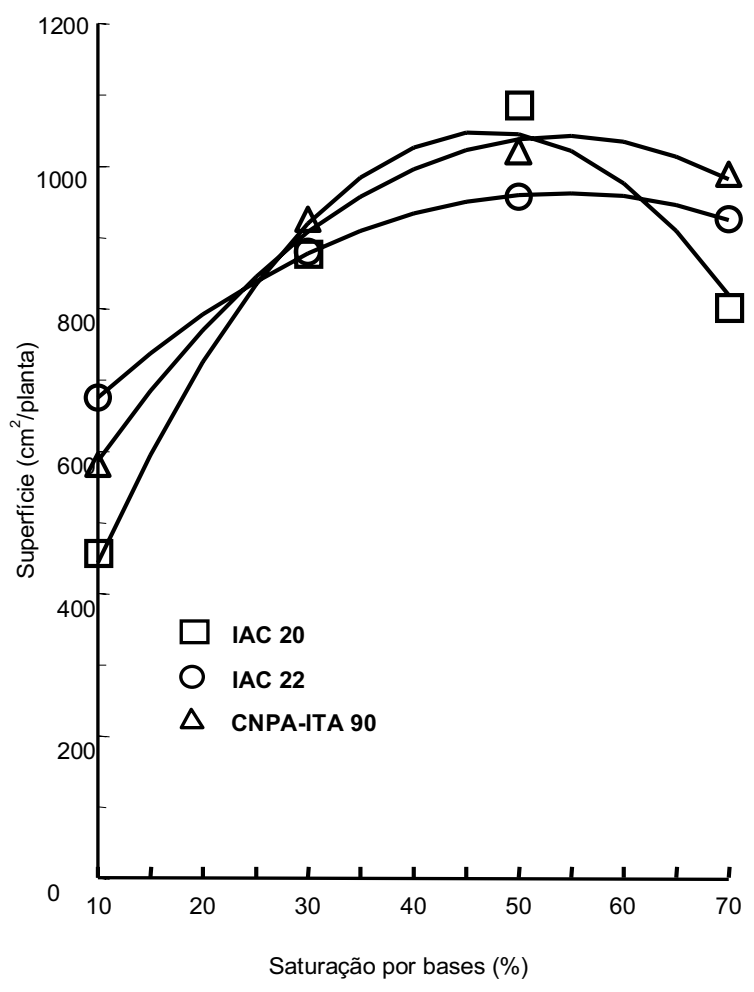

FIG. 2. Comprimento e superfície de raízes de algodoeiro em razão da cultivar e da saturação do solo por bases. Comprimento: IAC $20-y=3,90+2,41 x-0,0238 x^{2}, R^{2}=0,99$; IAC $22-y=12,03+11,98 x^{1 / 2}-0,69 x, R^{2}=0,99 ;$ CNPA-ITA $90-y=18,63+1,71 x-0,152 x^{2}, R^{2}=0,99$.

Pesq. agropec. bras., Brasília, v.35, n.4, p.827-833, abr. 2000 
TABELA 1. Teor de nutrientes nas raízes de algodoeiro, em razão da saturação do solo por bases ${ }^{1}$.

\begin{tabular}{ccccc}
\hline Saturação & $\mathrm{Mg}$ & $\mathrm{B}$ & $\mathrm{Mn}$ & $\mathrm{Zn}$ \\
\hline$(\%)$ & $\left(\mathrm{g} \mathrm{kg}^{-1}\right)$ & $-109 \mathrm{c}$ & $384 \mathrm{a}$ & $28,4 \mathrm{a}$ \\
10 & $0,54 \mathrm{c}$ & $120 \mathrm{c}$ & $178 \mathrm{~b}$ & $26,8 \mathrm{~b}$ \\
30 & $1,16 \mathrm{~b}$ & $167 \mathrm{~b}$ & $140 \mathrm{c}$ & $25,5 \mathrm{bc}$ \\
50 & $1,61 \mathrm{a}$ & $195 \mathrm{a}$ & $110 \mathrm{~d}$ & $25,0 \mathrm{c}$ \\
\hline 70 & $1,72 \mathrm{a}$ & &
\end{tabular}

TABELA 2. Teor de nutrientes em algodoeiro, em razão da cultivar1.

\begin{tabular}{|c|c|c|c|c|}
\hline Cultivar & $\mathrm{Zn}$ na raiz & Mn na raiz & $\mathrm{K}$ na raiz & $\mathrm{K}$ na p. aérea \\
\hline & \multicolumn{2}{|c|}{ - $\left(\mathrm{mg} \mathrm{kg}^{-1}\right)$} & 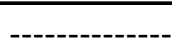 & 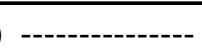 \\
\hline IAC 20 & $25,8 \mathrm{~b}$ & $186 \mathrm{c}$ & $12,4 \mathrm{a}$ & $8,18 b$ \\
\hline IAC 22 & $27,0 \mathrm{a}$ & $210 \mathrm{ab}$ & $11,0 \mathrm{ab}$ & $8,11 \mathrm{~b}$ \\
\hline CNPA-ITA 90 & $26,5 \mathrm{ab}$ & $212 \mathrm{a}$ & $9.7 \mathrm{~b}$ & $9,45 a$ \\
\hline
\end{tabular}

${ }^{1}$ Letras diferentes nas colunas indicam diferença significativa (LSD, $\mathrm{P}<0,05$ ).

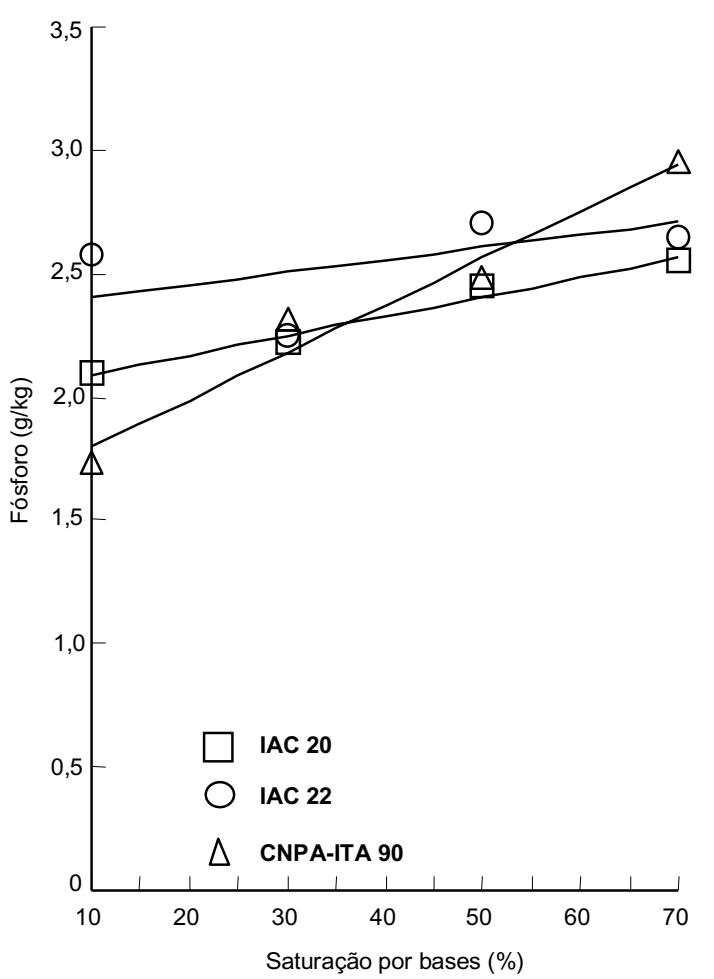

FIG. 3. Teor de fósforo na parte aérea do algodoeiro em razão da saturação do solo por bases e cultivares. IAC $20-\mathrm{y}=\mathbf{2 , 0 1}+\mathbf{0 , 0 0 7 9 9 x}$, $\mathbf{R}^{2}=0,99 ;$ IAC $22-\mathbf{y}=2,35+0,0052 x$, $R^{2}=0,89$; CNPA-ITA $90-y=1,606+0,0191 x$, $\mathbf{R}^{2}=\mathbf{0 , 9 8}$.
Foi ainda observado efeito das cultivares sobre os teores de K (Tabela 2) na parte aérea das plantas, sem efeito significativo das saturações do solo por bases. A cultivar CNPA-ITA 90 mostrou-se mais exigente em $\mathrm{K}$ que as demais, mas é interessante notar que a translocação de $\mathrm{K}$ nessa cultivar é mais eficiente, uma vez que mostrou menor teor do nutriente nas raízes e maior teor na parte aérea que as cultivares IACs.

Foi verificado efeito significativo da saturação por bases nos teores de $\mathrm{Ca}, \mathrm{Mg}$ e $\mathrm{Mn}$ (Fig. 4), porém não houve efeito de cultivares sobre esses parâmetros, e, tampouco, interação significativa. Entretanto, como esperado, houve aumento nos teores de $\mathrm{Ca}$ e $\mathrm{Mg}$ na parte aérea em resposta ao aumento na saturação por bases, ao mesmo tempo em que os teores de Mn foram diminuídos. Utilizando-se as equações ajustadas, as máximas produções de matéria seca ocorreram com 63,70 e $44 \%$ de saturação por bases, respectivamente para IAC 20, IAC 22 e CNPA-ITA 90. Essas saturações correspondem a 69, 60 e $126 \mathrm{mg} \mathrm{kg}^{-1}$ de $\mathrm{Mn}$ na parte aérea, respectivamente de IAC 20, IAC 22 e CNPA-ITA 90. É interessante notar que CNPA-ITA 90 atingiu a máxima produtividade com teores de $\mathrm{Mn}$ acima de $120 \mathrm{mg} \mathrm{kg}^{-1}$ na parte aérea, enquanto as IACs somente atingiram a máxima produtividade com 


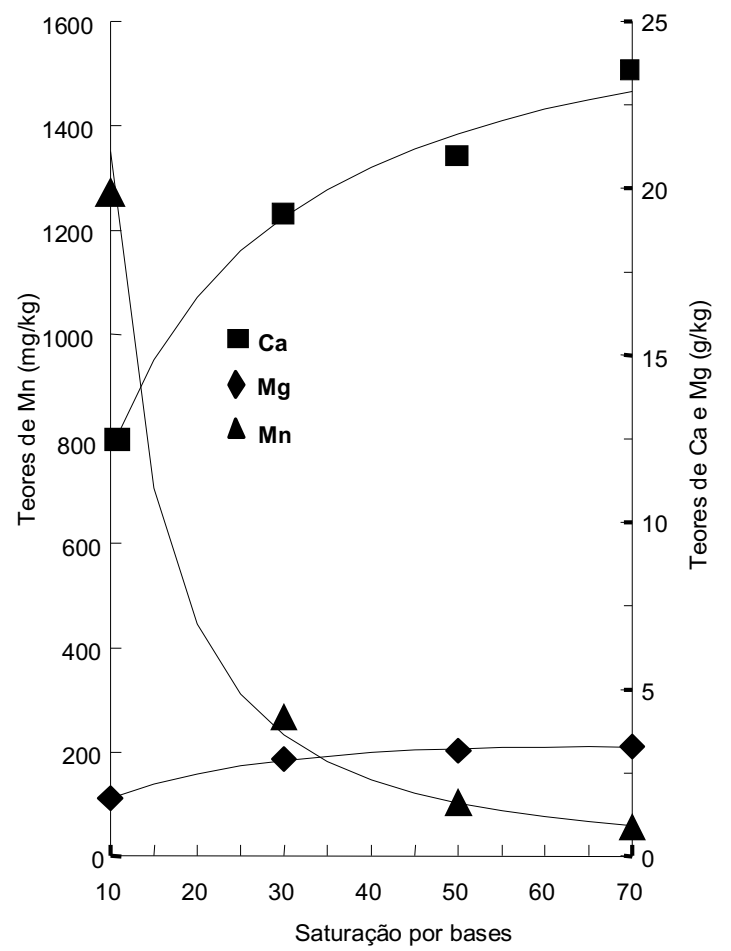

FIG.4. Teor de Ca, Mg e Mn na parte aérea do algodoeiro em razão da saturação do solo por bases. Média de três cultivares. $\mathrm{Ca}-\mathrm{y}=\mathbf{2 6 , 9 x} /(12,1+\mathbf{x}), \mathbf{R}^{2}=0,99$;

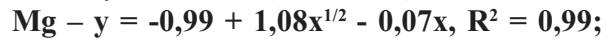
$M n-y=54133 x^{-1,603}, R^{2}=0,99$.

teores de Mn menores que $70 \mathrm{mg} \mathrm{kg}{ }^{-1}$. Como nas saturações por bases mais altas houve prejuízo na produção de matéria seca de CNPA-ITA 90, pode-se inferir que este material é menos sensível à toxicidade de Mn que as IACs.

Quanto aos outros nutrientes na parte aérea, não foi observado efeito significativo dos tratamentos.

Com relação à absorção de nutrientes por unidade de comprimento de raiz, notou-se resposta significativa apenas em função das saturações por bases no tocante a P, Ca, Mg e Mn. A absorção de P, Ca e $\mathrm{Mg}$ foi aumentada com o aumento na saturação do solo por bases (Tabela 3). Como não houve efeito das saturações por bases nos teores desses nutrientes na parte aérea da planta, é possível inferir que na menor saturação houve deficiência dos nutrientes no solo, que não permitia uma velocidade de supri-
TABELA 3. Quantidade de nutrientes absorvidos por metro de raiz de algodoeiro, em razão da saturação do solo por bases ${ }^{1}$.

\begin{tabular}{ccccc}
\hline Saturação & $\mathrm{P}$ & $\mathrm{Ca}$ & $\mathrm{Mg}$ & $\mathrm{Mn}$ \\
\hline$(\%)$ & $-0-176 \mathrm{~b}$ & $774 \mathrm{c}$ & $110 \mathrm{~b}$ & $80,6 \mathrm{a}$ \\
10 & $198 \mathrm{ab}$ & $1259 \mathrm{~b}$ & $207 \mathrm{a}$ & $20,4 \mathrm{~b}$ \\
30 & $188 \mathrm{ab}$ & $1280 \mathrm{~b}$ & $208 \mathrm{a}$ & $8,4 \mathrm{c}$ \\
50 & $208 \mathrm{a}$ & $1557 \mathrm{a}$ & $235 \mathrm{a}$ & $5,3 \mathrm{c}$ \\
70 & \multicolumn{4}{c}{}
\end{tabular}

mento para manter o crescimento da planta, uma vez que houve resposta da planta à calagem, em termos de produção de matéria seca. Com a correção do solo, as raízes começaram a absorver os nutrientes em maior velocidade, suprindo a necessidade das plantas. $\mathrm{Na}$ maior saturação, com prejuízo no comprimento e superfície radiculares, há um aumento nas quantidades absorvidas por unidade de comprimento radicular, que, no caso, foi suficiente para manter um bom crescimento nas cultivares IAC 20 e IAC 22.

\section{CONCLUSÕES}

1. A calagem é necessária para que ocorra bom crescimento radicular do algodoeiro.

2. A cultivar IAC 22 tem sistema radicular mais tolerante à baixa saturação do solo por bases, mas o máximo crescimento radicular é observado em saturação do solo por bases ao redor de $50 \%$, nas três cultivares.

3. A cultivar CNPA-ITA 90 é mais exigente em K e mais tolerante ao Mn, e sofre prejuízo na produção de matéria seca da parte aérea quando a saturação do solo por bases se aproxima de $70 \%$.

4. Com relação ao P, CNPA-ITA 90 mostra-se menos eficiente que as demais na absorção em baixas saturações por bases e mais eficiente em solo corrigido.

\section{REFERÊNCIAS}

ADAMS, F.; MOORE, B.L. Chemical factors affecting root growth in subsoil horizons of coastal plain soils. Soil Science Society of America. Journal, Madison, v.47, p.99-102, 1983. 
ADAMS, F.; PEARSON, R.W. Differencial response of cotton and peanuts to subsoil acidity. Agronomy Journal, Madison, v.62, p.9-12, 1970.

BROUDER, S.M.; CASSMAN, K.G. Root development of two cotton cultivars in relation to potassium uptake and plant growth in vermiculite soil. Field Crops Research, Amsterdam, v.23, p.187-203, 1990.

HALLMARK, W.B.; BARBER, S.A. Root growth and morphology, nutrient uptake and nutrient status of early grown soybeans as affected by soil $\mathrm{P}$ and $\mathrm{K}$. Agronomy Journal, Madison, v.76, p.209-212, 1984

MARTINI, J.A.; MUTTERS, R.G. Soybean root growth and nutrient uptake as affected by lime rates and plant age. I. Al, Mn, P and S. Turrialba, San José, v.389, p.1-8, 1989.

NAYAKEKORALA, H.; TAYLOR, H.M. Phosphorus uptake rates of cotton roots at different growth stages from different soil layers. Plant and Soil, Dordrecht, v.122, p.105-110, 1990.

RAIJ, B. van; QUAGGIO, J.A. Métodos de análise de solo para fins de fertilidade. Campinas : Instituto Agronômico, 1983. 31p. (Boletim técnico, 81).

ROSOLEM, C.A.; ASSIS, J.S.; SANTIAGO, A.D. Root growth and mineral nutrition of corn hybrids as affected by phosphorus and lime. Communications in Soil Science and Plant Analysis, New York, v.25, p.249-254, 1994.

ROSOLEM, C.A.; BICUDO, S.J.; MARUBAYASHI, O.M. Soybean yield and root growth as affected by lime rate and quality. In: DATE, R.A.; GRUNDON, N.J.; RAYMENT, G.E.; PROBERT, M.E. (Eds.). Plant soil interactions at low pH: Principles and management. Dordrech: Kluwer, 1995. p.543-547.

ROSOLEM, C.A.; SCHIOCHET, M.A.; SOUSA, L.S.; WITACKER, J.P.T. Root growth and cotton nutrition as affected by liming and soil compaction. Communications in Soil Science and Plant Analysis, New York, v.29, p.169-177, 1998.

SILVA, N.M.; CARVALHO, L.H.; QUAGGIO, J.A. Ensaio de longa duração com calcário e cloreto de potássio na cultura do algodoeiro. Bragantia, Campinas, v.54, p.353-360, 1995.

TAYLOR, D.; ARKIN, G.F. Root zone modification fundamentals and alternatives. In: TAYLOR, H.M.; ARKIN, G.F. (Eds.). Modifying the root environment to reduce crop stress. St. Joseph : ASAE, 1981. p.3-16.

TENNANT, D. A test of a modified line intersect method of estimating root length. Journal of Ecology, London, v.63, p.995-1001, 1975. 